\title{
Voltage Stability of Distributed Generators by means of Discrete Abstraction*
}

\author{
Marjorie Cosson ${ }^{1,3}$, Hervé Guéguen ${ }^{2}$, Didier Dumur ${ }^{1}$, Cristina Stoica Maniu ${ }^{1}$, \\ Vincent Gabrion ${ }^{3}$ and Gilles Malarange ${ }^{3}$
}

\begin{abstract}
Among the consequences of massive distributed generation development, voltage rise is a major concern. The local reactive power control laws considered to solve this issue might lead to voltage instability. In this context, the current paper proposes a methodology to study voltage stability of a distribution feeder hosting distributed generation. The approach relies on the construction of an equivalent discrete abstraction of the continuous transition system by bisimulation calculation. The stability of the system is assessed conducting a reachability analysis of the equivalent discrete abstraction. An illustrative application of the proposed approach to a real medium-voltage 124 buses network with a single distributed generator reveals unstable operating regions of the network and helps designing a measurement filter ensuring system stability without loss of rapidity.
\end{abstract}

\section{INTRODUCTION}

With the increasing number of Distributed Generators (DGs), distribution networks have seen their power flows substantially modified over the past decade [1] - [3]. One of the consequences is the increase of the voltage along distribution feeders hosting generation. To be able to maintain the voltage within specified limits while avoiding network reinforcement costs, several solutions have been investigated in the literature [4] - [6]. Among these ideas, local control laws of DGs reactive power $(Q)$ as a function of their voltage $(U)$ have been considered.

Preliminary studies conducted by a French distribution system operator [7] - [9] have led to choose the shape of $Q(U)$ law shown by Fig. 1 along with a discrete measurement filter. In [9], the impact of one $Q(U)$ law on existing grid assets - such as On-Load Tap Changers (OLTC) or capacitor banks - is studied. This experimentation concludes that $Q(U)$ law might interact with substation equipment increasing the occurrence of material solicitation and so

\footnotetext{
*This study has been carried out in the RISEGrid Institute (www.supelec.fr/342_p_38091/risegrid-en.html), joint program between CentraleSupelec and EDF ('Electricité de France') on smarter electric grids.

${ }^{1}$ Marjorie Cosson, Didier Dumur and Cristina Stoica Maniu are with Laboratoire des Signaux et Systèmes (L2S, UMR 8506 CentraleSupelec - CNRS - Université Paris-Sud), Automatic Control Department, 3 rue Joliot Curie, 91192 Gif-surYvette, France, \{marjorie.cosson; didier.dumur; cristina.stoica\} ecentralesupelec.fr

${ }^{2}$ Hervé Guéguen is with CentraleSupelec-IETR, Avenue de la Boulaie, CS47601, F-35576 Cesson Sévigné Cedex, France, herve.gueguen@centralesupelec.fr

${ }^{2}$ Gilles Malarange and Vincent Gabrion are with EDF R\&D, Department Economic and Technical Analysis of Energy Systems (EFESE), F-92410 Clamart, France, \{gilles.malarange; vincent.gabrion\}@edf.fr
}

causing technical fatigue depending on control law parameters. Other experimental investigations have been conducted to study interactions between several DGs of the same feeder [10], [11]. Indeed, interactions between $Q(U)$ law and existing control laws - such as OLTC or capacitor banks - can appear causing voltage instability. In this context, a formal analysis is necessary to generalize the empirical results on the stability of networks involving one or several DGs equipped with $Q(U)$ laws.

In this paper, a methodology is developed to study voltage stability of distribution feeders with $Q(U)$ laws. Due to the nonlinearity of the $Q(U)$ law, classical stability analysis cannot be used here. To assess system stability, the system is modeled as a continuous transition system using hybrid system tools [12]. Because of the infinite number of possible operating voltages, unsafe regions are still difficult to study. Starting from a continuous system, the authors of [12] propose an equivalent finite discrete transition system which simplifies the analysis of safe operations.

The aim of this paper is to use discrete abstraction techniques to construct a finite discrete transition system equivalent to the continuous model of a medium-voltage (MV) feeder connecting a single distributed generator. The main contribution consists in developing a method to conduct a formal stability analysis of the system allowing to set measurement filter and $Q(U)$ law parameters in order to ensure voltage stability.

First of all, the system modeling as a continuous transition system is presented in Section II. Then, Section III details the construction of the equivalent discrete transition system and the study of its stability. In Section IV, this method is applied to a real case study. System stability is discussed and a measurement filter ensuring system stability is proposed. Conclusions and perspectives are presented in Section V.

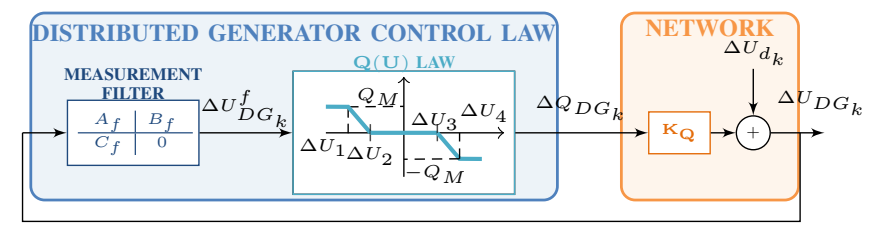

Fig. 1. Proposed closed-loop model for the voltage stability study 


\section{SYSTEM MODELING}

\section{A. System Description}

A medium-voltage feeder connecting several consumers and a single distributed generator equipped with the $Q(U)$ law presented in Fig. 1 is modeled to study the possible voltage instability caused by this control law. Such phenomena are generally slower than the DG control law sample time [13]. As the DG sample time is about one second, unstable phenomena would have a time constant from a few seconds to a few tens of seconds. Thus the study of these phenomena does not require to model transient states of faster phenomena. As network lines transients are electromagnetic phenomena, their dynamics are faster than one second [14]. So they are modeled in steady-state. By sensitivity analysis, the variation of DG voltage magnitude can be expressed as a function of the variations of controlled and uncontrolled variables [5]. In the studied case, the only controlled variable is the DG reactive power $\left(Q_{D G}\right)$. Other variables (such as DG active power, active and reactive consumption or busbar voltage) will be treated as disturbances $\left(\underline{U}_{d}\right)$. The magnitude of a complex $\underline{Z}$ will be denoted $Z$. The linearized DG voltage magnitude deviation $\Delta U_{D G}$ can be expressed as follows [5]:

$$
\Delta U_{D G}=K_{Q} \Delta Q_{D G}+\Delta U_{d},
$$

where $K_{Q}$ is the sensitivity coefficient of DG's voltage magnitude deviation to control variable variation $\left(\Delta Q_{D G}\right)$ and $\Delta U_{d}$ represents the disturbance variations magnitude modeling the impact of the uncontrolled variables variations.

The DG is supposed to be connected to the grid through power electronics as most of the DGs are [13]. As power electronics reach steady-state in less than one second [13], they are also modeled in steady-state. To conclude, the only dynamics considered are the DG measurement filter dynamics.

\section{B. Closed-Loop System}

As described in the previous sub-section, the MV-feeder hosting a single DG equipped with $Q(U)$ law can be modeled in closed-loop as represented in Fig. 1.

In order to study the impact of the filter on the stability of a MV-feeder, a generic discrete time-invariant statespace model $\left(A_{f}, B_{f}, C_{f}, 0\right)$ is chosen to describe the measurement filter. In this model, $x_{k} \in \mathbb{X}_{x} \subseteq \mathbb{R}^{N_{x}}$ - with $N_{x}$ the dimension of the vector $x-$ is the state vector, $\Delta U_{D G_{k}} \in \mathbb{R}$ is the input and $\Delta U_{D G_{k}}^{f} \in \mathbb{R}$ is the output.

$$
\left\{\begin{array}{c}
x_{k+1}=A_{f} x_{k}+B_{f} \Delta U_{D G_{k}} \\
\Delta U_{D G_{k}}^{f}=C_{f} x_{k}
\end{array}\right.
$$

The DG control law is a discrete-time law with a sample time $T_{S}$ of one second. The $Q(U)$ law considered in this paper is not linear but it can be noticed that it is piecewise linear. The $Q(U)$ law can be represented by:

$$
\Delta Q_{D G_{k}}=\alpha_{i} \Delta U_{D G_{k}}^{f}+\beta_{i} \text { if } \Delta U_{i-1} \leq \Delta U_{D G_{k}}^{f} \leq \Delta U_{i},
$$

for all $i$ in $\{1, \ldots, 5\}$. Please note that $\Delta U_{0}$ and $\Delta U_{5}$ are respectively defined by $\Delta U_{D G_{\min }}$ and $\Delta U_{D G_{\max }}$.
For instance, if the output of the filter $\left(\Delta U_{D G_{k}}^{f}\right)$ is greater than $U_{4}$, then the reactive power variation is determined by the equation $\Delta Q_{D G_{k}}=-Q_{M}$, which represents the maximum of reactive power the DG can absorb from the network (see Fig. 1), thus $\alpha_{5}=0$ and $\beta_{5}=-Q_{M}$. The network is modeled by (1) and so it is represented by the gain $K_{Q}$. To represent both the filter state $\left(x_{k} \in \mathbb{X}_{x}\right)$ and the network influence $\left(\Delta U_{d_{k}} \in \mathbb{X}_{U_{d}}\right)$, an extended discrete time state-space representation is chosen introducing a new state vector $\mathcal{X}_{k}=\left[\begin{array}{cc}x_{k}^{T} & \Delta U_{d_{k}}\end{array}\right]^{T} \in \mathbb{X} \subset \mathbb{R}^{N_{x}+1}$. Please note that network dynamics are neglected: $\Delta U_{d_{k+1}}=\Delta U_{d_{k}}=\Delta U_{d}$.

The continuous transition system associated to the closedloop system is composed of five modes each of them associated with constraints and a linear dynamics. For instance, the fifth mode constraints $K_{5} \mathcal{X}_{k} \leq L_{5}$ are obtained from:

$$
\left\{\Delta U_{4} \leq C_{f} x_{k}\right\} \cap\left\{x_{k} \in \mathbb{X}_{x}\right\} \cap\left\{\Delta U_{d_{k}} \in \mathbb{X}_{U_{d}}\right\}
$$

The linear dynamics of the fifth mode is obtained from Fig. 1:

$$
\left\{\begin{array}{l}
x_{k+1}=A_{f} x_{k}+B_{f} \Delta U_{D G_{k}} \\
\Delta U_{D G_{k}}=K_{Q} \Delta Q_{D G_{k}}+\Delta U_{d_{k}} \\
\Delta Q_{D G_{k}}=\alpha_{5} \Delta U_{D G_{k}}^{f}+\beta_{5} \\
\Delta U_{D G_{k}}^{f}=C_{f} x_{k}
\end{array}\right.
$$

From (4), the linear dynamics of the extended state vector $\mathcal{X}_{k}$, in the fifth mode, can be written as follows:

$$
\mathcal{X}_{k+1}=\underbrace{\left[\begin{array}{cc}
A_{f}+\alpha_{5} K_{Q} B_{f} C_{f} & B_{f} \\
0 & 1
\end{array}\right]}_{\mathcal{A}_{5}} \mathcal{X}_{k}+\underbrace{\left[\begin{array}{c}
\beta_{5} K_{Q} B_{f} \\
0
\end{array}\right]}_{\mathcal{F}_{5}} .
$$

To conclude, the continuous transition system can be expressed by:

$$
\mathcal{X}_{k+1}=\mathcal{A}_{i} \mathcal{X}_{k}+\mathcal{F}_{i} \text { if } K_{i} \mathcal{X}_{k} \leq L_{i} \forall i=1, \ldots, 5
$$

The purpose of this study is to assess voltage stability of this continuous transition discrete-time system. A stable system is considered to be a system reaching steady-state after any variation of the perturbations $\Delta U_{d_{k}} \in X_{U_{d}}$. So, to check system stability, a reachability analysis from any initial condition is necessary to verify that the perturbation does not end into a cycling trajectory. From the continuous transition system described by (6), reachability computations cannot be carried out. In order to be able to study stability, a finite discrete abstraction of this continuous transition discrete-time system is developed in the next section.

\section{EQUIVALENT DISCRETE ABSTRACTION AND STABILITY ANALYSIS}

\section{A. Sufficient Discrete Abstraction}

In this subsection, a discrete transition system is abstracted from the continuous transition system described by (6). First, five discrete states $\left(\mathfrak{M}_{i}^{0}\right)_{i=1, \ldots, 5}$ are constructed from the continuous transition system. Each discrete state $\mathfrak{M}_{i}^{0}$ corresponds to a domain $D_{i}^{0}$ of the continuous state-space $(\mathbb{X})$ defined by the corresponding constraints:

$$
D_{i}^{0}=\left\{\forall s \in \mathbb{X}: K_{i} s \leq L_{i}\right\},
$$


and a linear dynamics defined by $\mathcal{A}_{i}$ and $\mathcal{F}_{i}$ from (6). Let note $\mathbb{D}^{0}$ the set of domains $\left(D_{i}^{0}\right)_{i=1, \ldots, 5}$. A discrete transition between $\mathfrak{M}_{i}^{0}$ and $\mathfrak{M}_{j}^{0}$ is possible if it exists $s \in D_{i}^{0}$ such as after applying $\mathfrak{M}_{i}^{0}$ dynamics to $s$, the domain $D_{j}^{0}$ is reached, i.e. $\mathcal{A}_{i} s+\mathcal{F}_{i} \in D_{j}^{0}$.

So far, it is considered that, from each of the five discrete modes, a transition toward every discrete mode is possible. Thus, the discrete abstraction can be represented as a graph with a five number of nodes (corresponding to the discrete states $\left.\left(\mathfrak{M}_{i}^{0}\right)_{i=1, \ldots, 5}\right)$ and edges (corresponding to transitions). This discrete automaton is represented in Fig. 2.

Stability is studied by reachability analysis which is easier to conduct on the discrete abstraction than on the continuous transition system as the number of discrete states is finite and the discrete trajectories are obtain by the discrete transitions.

In this initial discrete abstraction, all discrete trajectories have been considered as possible without verifying if it actually exists $s \in \mathbb{X}$ verifying it. Thus all continuous trajectories are represented by a discrete trajectory but all discrete trajectories do not correspond to a continuous trajectory. The discrete abstraction is said to be sufficient but not equivalent to the continuous transition system. Thus, stability of the discrete abstraction implies stability of the continuous transition system but instability of the discrete system does not necessarily imply instability of the continuous transition system. To be able to conclude on continuous system stability, the sufficient discrete abstraction must be stable.

In this case, it can be seen in Fig. 2 that there exist discrete transitions corresponding to cycles and thus unstable discrete trajectories. For instance, a discrete cycle exists between $\mathfrak{M}_{1}^{0}$ and $\mathfrak{M}_{3}^{0}$ but it is not proven yet that the subset of $D_{1}^{0}$ leading to $D_{3}^{0}$ can be reached from $D_{3}^{0}$. To be able to conclude on system stability, the subsets of $\mathbb{D}^{0}$ have to be divided according to their successor in order to remove from the graph discrete trajectories which do not correspond to continuous ones.

These calculations are done for every discrete state of the sufficient discrete abstraction until a discrete abstraction equivalent to the continuous transition system is found. Section IV illustrates this on a simple 2-dimensional example.

\section{B. Equivalent Discrete Abstraction}

The discrete abstraction is equivalent to the continuous transition system if every discrete transition corresponds to

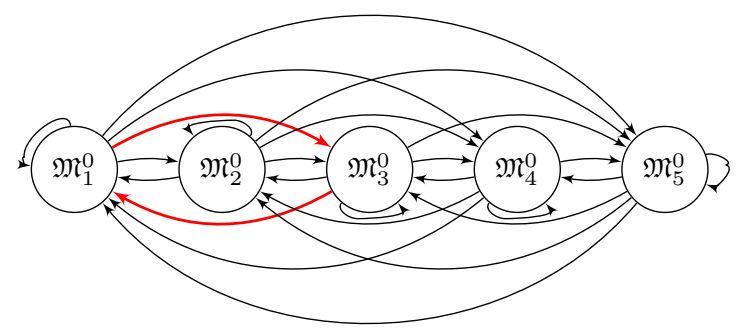

Fig. 2. Discrete automaton associated with the initial discrete abstraction a continuous one. Then, stability of the discrete abstraction is equivalent to continuous transition system stability. As in practice, only one transition is possible from a given $s \in$ $\mathbb{X}$, the equivalent discrete abstraction is obtained when each discrete state corresponds to a single transition, i.e. if it exists a transition between two discrete states $\mathfrak{M}_{i}$ and $\mathfrak{M}_{j}$, then, for all $s \in D_{i}, A_{i} s+F_{i} \in D_{j}$.

To obtain this equivalent abstraction, bisimulation calculations are applied [15]. The main idea of this method is to test, for all $i=1, \ldots, 5$, if it exists $\left(s_{1}, s_{2}\right) \in\left(D_{i}^{0}\right)^{2}$ such as after applying the dynamics associated with $D_{i}^{0}$, two different domains of $\mathbb{D}^{0}$ are reached. If so, $D_{i}^{0}$ is divided according to its destinations. To perform this study, the predecessor of a domain $D_{i}^{0} \subset \mathbb{D}^{0}$ is defined as follows:

$$
\operatorname{Pre}\left(D_{i}^{0}\right)=\left\{s \in \mathbb{X} \mid\left(\mathcal{A}_{s} s+\mathcal{F}_{s}\right) \in D_{i}^{0}\right\}
$$

For every pair of subsets $\left(D_{i}^{0}, D_{j}^{0}\right)$ of $\left(\mathbb{D}^{0}\right)^{2}, D_{j}^{0} \cap \operatorname{Pre}\left(D_{i}^{0}\right)$ is compared to $D_{j}^{0}$. If they are equal then all $s \in D_{j}^{0}$ leads to $D_{i}^{0}$, if they are disjoint, no discrete state of $D_{j}^{0}$ leads to $D_{i}^{0}$. Otherwise, part of $D_{j}^{0}\left(D_{j i}^{1}\right)$ leads to $D_{i}^{0}$ and the rest $\left(D_{j \bar{i}}^{1}\right)$ leads elsewhere.

$$
D_{j i}^{1}=D_{j}^{0} \cap \operatorname{Pre}\left(D_{i}^{0}\right) \text { and } D_{j \bar{i}}^{1}=D_{j}^{0} \backslash D_{j i}^{1}
$$

So if $D_{j}^{0}$ has several destinations, the bisimulation algorithm divides it into two subsets: one with a single destination $\left(D_{j i}^{1}\right)$ and one with potentially several destinations $\left(D_{j \bar{i}}^{1}\right)$. If so, $D_{j \bar{i}}^{1}$ is divided again until it can be expressed as the union of domains, all of them being equal to the predecessor of another subset of $\mathbb{D}^{0}$. This partition is done for every domain of $\mathbb{D}^{0}$ and the new set of domains is called $\mathbb{D}^{1}$. To each domain $D_{i}^{1} \subset \mathbb{D}^{1}$, a discrete state $\mathfrak{M}_{i}^{1}$ is associated. A discrete transition from $\mathfrak{M}_{i}^{1}$ to $\mathfrak{M}_{j}^{1}$ is created if it exists $s \in D_{i}^{1}$ such as $\mathcal{A}_{i} s+\mathcal{F}_{i} \in D_{j}^{1}$. the domains composing $\mathbb{D}^{1}$ might need to be divided again if a discrete state leads to several discrete states. For instance, if $D_{i}^{0}$ has been divided, then $D_{j i}^{1}$ will need to be divided again.

This calculation ends when all discrete states of the abstraction correspond to a single transition, then, the set of continuous space domains is called $\mathbb{D}^{B}$. It satisfies:

$$
\forall\left(D, D^{\prime}\right) \in\left(\mathbb{D}^{B}\right)^{2}, D \cap \operatorname{Pre}\left(D^{\prime}\right)=\varnothing \vee D \cap \operatorname{Pre}\left(D^{\prime}\right)=D
$$

\section{Stability Analysis}

To study the continuous transition system stability, reachability analysis is conducted on the equivalent discrete abstraction. Let us note Post $(D)$ the domain of successors of a domain $D$. $\operatorname{Post}^{n}(D)$ represents the continuous states of $\mathbb{X}$ reachable from $D$ in $n \in \mathbb{N}$ transitions. A domain $D \in \mathbb{X}$ is considered stable if every continuous trajectory starting from $D$ reaches, after a given number $n$ of transitions, an invariant domain which can be written:

$$
\exists n \in \mathbb{N}, \text { Post }^{n+1}(D) \subseteq \operatorname{Post}^{n}(D)
$$

Coming back to the studied system, the continuous transition system is stable if none of the disturbances $\Delta U_{d} \in \mathbb{X}_{U_{d}}$ corresponds to a trajectory ending into a cycle. The system 
is stable if each $D_{i}^{b} \in \mathbb{D}^{B}$ satisfies (11). The reachability analysis is carried out on the equivalent discrete abstraction constructed by bisimulation. As each discrete state of the equivalent discrete abstraction is the origin of a single discrete transition, the successor of the corresponding continuous state-space domain is already calculated. So following the discrete transitions, discrete paths are studied.

To conclude, the nonlinear dynamics of the $Q(U)$ law chosen leads to model the system as a continuous transition system (6) upon which no formal stability analysis can be carried out. To overcome this issue, an equivalent discrete abstraction is constructed by bisimulation calculation. Thus, stability of the continuous transition system can be equivalently assessed by conducting reachability analysis on the equivalent discrete abstraction. This method proposes the formal stability analysis of a feeder knowing its topology $\left(K_{Q}\right)$, filter and $Q(U)$ law parameters assuming that the bisimulation calculation converges.

\section{CASE-STUDY: STABILITY OF A REAL MEDIUM VOLTAGE FEEDER AND DISCUSSION ON FILTER DESIGN}

\section{A. Real Case-Study Description}

The considered system is a real MV-feeder of the ERDF ("Électricité Réseau Distribution France") network. It is exploited at $20 \mathrm{kV}$ and contains approx $20 \mathrm{~km}$ of aerial distribution lines. This feeder hosts about $300 \mathrm{~kW}$ of consumption and a single DG: a wind farm of $5 \mathrm{MW}$. This DG is considered to be equipped with the control law presented in Fig. 1. The goal is to study its intrinsic stability and the impact of the filter on system stability and rapidity. To discuss intrinsic stability, preliminary calculations are done considering a pure delay filter. It can be described by:

$$
\left\{\begin{array}{c}
x_{k+1}=\Delta U_{D G_{k}} \\
\Delta U_{D G_{k}}^{f}=x_{k}
\end{array} .\right.
$$

So the corresponding state-space model is $(0,1,1,0)$. The dimension $\left(N_{x}\right)$ of the filter state-space vector is one. So the extended system state-space dimension will be two. This allows us to represent subsets of the discrete state-space in the 2-dimensional plane $\left(x_{k}, \Delta U_{d_{k}}\right)$. These variables satisfy the following constraints:

$$
\forall k \in \mathbb{N},\left\{\begin{array}{l}
x_{k} \in\left[\Delta U_{D G_{\min }} ; \Delta U_{D G_{\max }}\right] \\
\Delta U_{d_{k}} \in\left[\Delta U_{d_{\min }} ; \Delta U_{d_{\max }}\right]
\end{array} .\right.
$$

For instance, the fifth mode constraints obtained in (3) can be written:

$$
\left\{\begin{array}{l}
\Delta U_{4} \leq x_{k} \\
\Delta U_{D G_{\min }} \leq x_{k} \\
x_{k} \leq \Delta U_{D G_{\max }} \\
\Delta U_{d_{\min }} \leq \Delta U_{d_{k}} \\
\Delta U_{d_{k}} \leq \Delta U_{d_{\max }}
\end{array} \Leftrightarrow K_{5} \mathcal{X}_{k} \leq L_{5} .\right.
$$

Physical limits of the network are represented by the minimum and maximum DG variations $\left(\Delta U_{D G_{m i n}}\right.$ and $\Delta U_{D G_{\max }}$ ) which corresponds to voltages at which the network is not supposed to be operated. To avoid operations outside of these limits, circuit breakers automatically open the circuit in which the fault has been detected. In the case of this $20 \mathrm{kV}$ network, $\Delta U_{D G_{\max }}$ and $\Delta U_{D G_{\min }}$ are set such as stability of the DG control law has to be ensured for operating points from $18 \mathrm{kV}$ to $22 \mathrm{kV}$.

So, the matrices $K_{5}$ and $L_{5}$ can be determined.

$$
K_{5}=\left[\begin{array}{rr}
-1 & 0 \\
1 & 0 \\
-1 & 0 \\
0 & 1 \\
0 & -1
\end{array}\right] ; L_{5}=\left[\begin{array}{c}
-\Delta U_{4} \\
\Delta U_{D G_{\max }} \\
-\Delta U_{D G_{\min }} \\
\Delta U_{d_{\max }} \\
-\Delta U_{d_{\min }}
\end{array}\right]
$$

In this case, the fifth mode closed-loop equation is:

$$
x_{k+1}=\Delta U_{d}-Q_{M} K_{Q} .
$$

This leads to define the continuous transition system fifth dynamics as follows:

$$
\mathcal{A}_{5}=\left[\begin{array}{ll}
0 & 1 \\
0 & 1
\end{array}\right] ; \mathcal{F}_{5}=\left[\begin{array}{c}
-Q_{M} K_{Q} \\
0
\end{array}\right] .
$$

This calculation can be done in every mode in order to make explicit the continuous transition model as described by (6). A formal stability analysis cannot be directly conducted on the continuous transition system. However, stability can be inferred from dynamical simulations.

In this case, it may be inferred with empirical simulations that the system is stable without a filter. This result can be verified from the reachability analysis of the equivalent discrete abstraction, as detailed in the next sub-section. Then, simulation results will be presented to illustrate the results.

\section{B. Stability Study}

1) Initial Discrete Automaton: From the continuous transition system, a discrete abstraction can be constructed. A discrete state is associated to each linear dynamics of the continuous transition system. Thus five discrete states $\left(\mathfrak{M}_{i}^{0}\right)_{i \in\{1, ., 5\}}$ and transitions in between them are defined such as explained previously. Fig 2 represents the discrete automaton associated with this discrete abstraction.

Fig. 3 (a) presents the initial partition $\left(D_{i}^{0}\right)_{i \in\{1, . ., 5\}}$ of the continuous state-space projected in the 2-dimensional space defined by the two components of the state-space vector. In Fig. 3 (a), the five domains $\left(D_{i}^{0}\right)_{i=1, . ., 5} \subset \mathbb{D}^{0}$ of the initial state-space division can be seen. For instance, the fifth domain is defined by (14). So it corresponds to every possible disturbance variation in $\mathbb{X}_{U_{d}}$ and a DG voltage variation in $\mathbb{X}_{x}$ and larger than $\Delta U_{4}$.

As it can be seen in Fig. 2, there exist cycles on this discrete abstraction. As the abstraction is not equivalent to the continuous system, the existence of discrete cycles does not prove anything about continuous transition system stability. To be able to conclude on system stability, at each iteration of the calculation, the subsets of the state-space leading to several destinations are divided using bisimulation calculation. The first iteration of this approach is detailed below. 

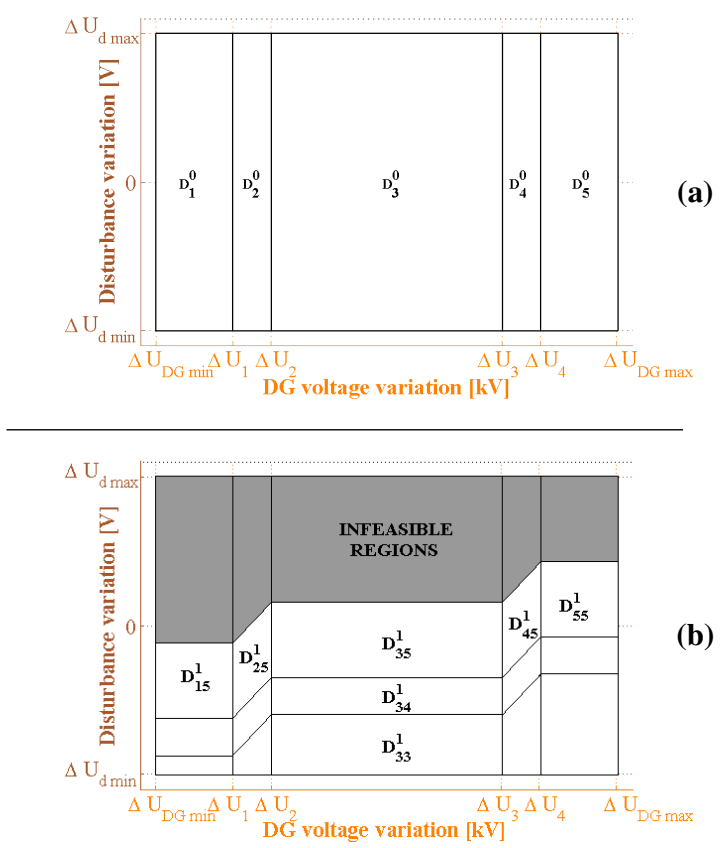

Fig. 3. (a) Initial discrete abstraction and (b) discrete abstraction after one iteration of the bisimulation calculation

2) First Iteration of the Bisimulation Calculation: As every discrete state $\mathfrak{M}_{i}^{0}$ corresponding to the continuous space domain $D_{i}^{0}$ is associated with five possible transitions, each domain $D_{i}^{0}$ will be divided into at most five subsets $\left(D_{i j}^{1}\right)_{i, j=1, \ldots, 5}$ defined by :

$$
D_{i j}^{1}=D_{i}^{0} \cap \operatorname{Pre}\left(D_{j}^{0}\right) \quad \forall i, j=1, \ldots, 5 .
$$

If a domain $D_{i j}^{1}$ is empty, then it means that no continuous transition corresponds to the discrete transition from $\mathfrak{M}_{i}^{0}$ to $\mathfrak{M}_{j}^{0}$. Fig. 3 (b) represents the partition of the state-space after the first iteration of the bisimulation calculation. As it can been seen on Fig. 3 (b), some regions of the state-space are infeasible. For the states in the shaded area of Fig. 3 (b), a given state $\mathcal{X}_{k} \in \mathbb{X}$ leads to - after the application of the corresponding dynamics - a state $\mathcal{X}_{k+1}$ outside the statespace $\mathbb{X}$. This corresponds to a voltage outside of the circuit breaker tolerance range so the circuit will be switched off by circuit breakers. Then these regions are considered infeasible.

At the end of the first iteration, the continuous state-space is divided into 15 subsets. It means that 10 of the 25 initial discrete transitions were not corresponding to a continuous transition. Some discrete states are still associated to several transitions as their destinations have been divided. More iterations of the bisimulation calculation are needed to obtain a discrete abstraction equivalent to the continuous system.

3) Reachability Analysis: After three iterations (see Fig. 4 (a)), only the crosshatched domains have several successors. Thus, trajectories which do not include one of these domains can be analyzed without further calculations. Indeed, such domains will not be divided in any further iteration of the calculation, so they belong $\mathbb{D}^{B}$. This is the case of the colored domains. For instance, $D_{1}^{B}$ leads entirely

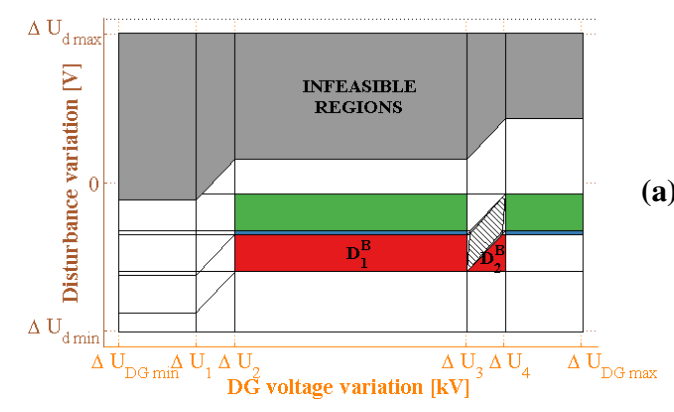

(a)

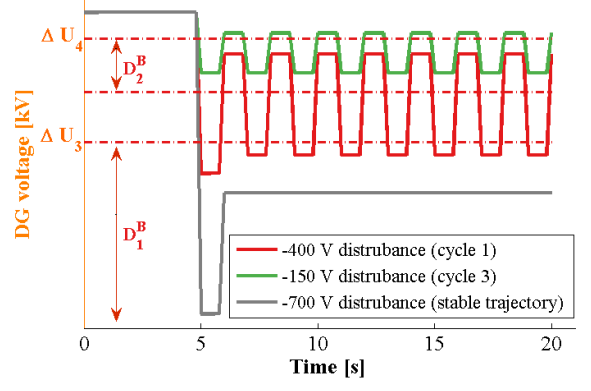

(b)

Fig. 4. (a) Results of the stability analysis in the state vector plane and (b) verification with dynamical analysis

to $D_{2}^{B}$ and $D_{2}^{B}$ leads entirely to $D_{1}^{B}$. So they will not be divided in any further iteration of the calculation, they belong to $\mathbb{D}^{B}$. So every trajectory leading to $D_{1}^{B}$ or $D_{2}^{B}$ ends into a cycle. The study of these domains reveals the existence of three cycles (colored domains of Fig.4). So, without reaching convergence, it can be concluded that the continuous transition system is unstable.

Unlike what was observed with the empirical study, the system appears to be unstable: cycles appear for $\Delta U_{d} \in$ $[-600 \mathrm{~V}, 0 \mathrm{~V}]$. Fig 4 (b) presents examples of dynamical simulations illustrating the unstable behavior of the system. The first trajectory is the response to a $-400 \mathrm{~V}$ disturbance step. According to Fig. 4 (a), it corresponds to a cycling trajectory in between two subsets: one corresponding to a voltage in $D_{1}^{B}$ and the other one in $D_{2}^{B}$. These results corresponds to the simulation example of Fig. 4 (b). The second trajectory shown by Fig. 4 (b) corresponds a cycling trajectory for a $\Delta U_{d}=-150 \mathrm{~V}$ in accordance with Fig. 4 (a). The third trajectory shown by Fig. 4 (b) corresponds to the initial empirical study showing stable behavior for $\Delta U_{d}=$ $-700 \mathrm{~V}$. Using this formal stability analysis, it is possible to conclude on continuous transition system stability.

\section{Discussion on Filter Parameters}

As this case study is unstable, the parameters of the DG control law have to be changed. Changing the $Q(U)$ law parameters will impact the DG control capability. Thus, whenever possible, it is preferable to adapt measurement filter parameters in order to ensure system stability without reducing its control capability. European standards [16] prescribe a first-order low-pass filter with a time constant between three to sixty seconds as measurement filter. For 

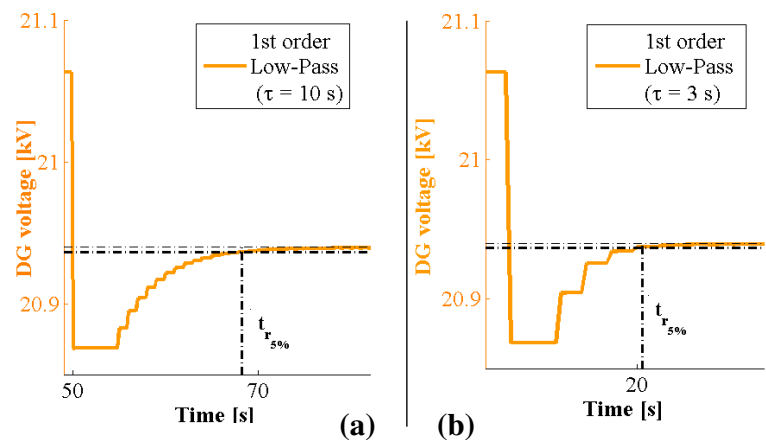

Fig. 5. Step response of the system including a first-order low-pass filter with a time constant of ten (a) and three (b) seconds

instance, Siemens inverters with a 10 -second time constant are studied in [17]. A discrete first-order low-pass filter with a 10 -second time constant is tested on this case-study. The formal analysis confirms stable operation with this filter. Fig. 5 (a) illustrates the response of the system for given initial conditions. It can be noticed that the $95 \%$ response time of the system is about 19 seconds.

With the formal stability analysis tool, a better trade-off between stability and rapidity can be found. Indeed, it is now possible to determine the filter smallest time constant ensuring system stability. Thus stability will be guaranteed with the minimum impact on system rapidity. In this case, the smallest prescribed time constant ( 3 seconds) is sufficient to ensure system stability. As it can be seen on Fig. 5 (b), the response time has been reduced from 19 seconds to 6 seconds. Please note that a first-order low-pass filter with a two-second time constant has also been tested and ensures system stability of this particular case-study.

To conclude, thanks to the formal analysis developed, the system stability can be assessed for all operating conditions. This allows to set measurement filter parameters in order to ensure system stability without slowing it down unnecessarily. Therefore, the DG control law is set to mitigate voltage deviation as fast as possible. In this particular case, a threesecond time-constant filter satisfies the requirements but this result is highly dependent on network parameters and DG installed capacity.

\section{CONCLUSIONS}

Due to massive insertion of distributed generators, the $Q(U)$ laws are expected to develop rapidly on distribution feeders. This multiplication might lead to voltage stability issues. As the proposed control law is nonlinear, its stability is difficult to study. The proposed methodology relies on the construction of an equivalent discrete abstraction of the continuous transition system. This abstraction is calculated using bisimulation algorithm. Then a reachability study allows to conclude on system stability. This method is illustrated on a real 124 nodes distribution feeder hosting one DG. The case study seems stable after a rapid empirical study. The proposed method reveals unstable operating regions.
A measurement filter is added to stabilize the system. The proposed method is also used to choose a filter ensuring system stability without degrading rapidity.

Further work will focus on the validation of this approach on several case studies. In particular, the choice of a firstorder low-pass filter and its time constant has to be investigated on various network architectures. Moreover, the impact of existing controls laws such as on-load tap-changers or capacitor banks should be studied. To do so, further work will be carried out including disturbances dynamics.

The main interest of the proposed approach is to extend it to feeders including multiple DGs. Indeed, as DGs multiply on feeders, stability issues will increase and particularly interactions in between DGs control laws might appear. An interesting challenge is to extend the proposed results for networks with several DGs per feeder while keeping acceptable computation load.

\section{REFERENCES}

[1] N. Hadjsaid, J.-F. Canard, and F. Dumas, "Dispersed generation impact on distribution networks," Computer Applications in Power, IEEE, vol. 12, pp. 22-28, Apr. 1999.

[2] C. Dai and Y. Baghzouz, "On the voltage profile of distribution feeders with distributed generation," in Proc. IEEE Power Engineering Society (PES) General Meeting, 2003.

[3] A. M. Azmy and I. Erlich, "Impact of distributed generation on the stability of electrical power system," in Proc. IEEE PES General Meeting, 2005.

[4] A. Allen, Y. Zhang, and B. Hodge, Voltage Impacts of Utility-Scale Distributed Wind. National Renewable Energy Laboratory, 2014.

[5] A. Borghetti, M. Bosetti, S. Grillo, S. Massucco, C. A. Nucci, M. Paolone, and F. Silvestro, "Short-term scheduling and control of active distribution systems with high penetration of renewable resources," Systems Journal, IEEE, vol. 4, no. 3, 2010.

[6] G. Valverde and T. Van Cutsem, "Model predictive control of voltages in active distribution networks," Smart Grid, IEEE Transactions on, vol. 4, no. 4, pp. 2152-2161, 2013.

[7] J. Duval, G. Delille, J.-L. Fraisse, and X. Guillaud, "Contribution of local voltage regulation to a better insertion of DG in distribution grids," CIRED, 2009.

[8] E. Chabod, L. Karsenti, J. Witkowski, and G. Malarange, "Local voltage regulation influence on DG and distribution network," in CIRED, 2012

[9] J. Witkowski, E. Lejay-Brun, G. Malarange, and L. Karsenti, "Field demonstration of local voltage regulation on ERDF MV network," CIRED, 2013.

[10] F. Beauné, A. Minaud, A. Pagnetti, G. Pelton, and L. Karsenti, "Voltage regulation on DG connected to MV network study and experimentations," in CIRED, 2014

[11] P. Esslinger and R. Witzmann, "Experimental study on voltage dependent reactive power control Q (V) by solar inverters in low-voltage networks," CIRED, 2013.

[12] R. Alur, T. A. Henzinger, G. Lafferriere, and G. J. Pappas, "Discrete abstractions of hybrid systems," Proc. of the IEEE, vol. 88, no. 7, 2000.

[13] J. Machowski, J. Bialek, and J. Bumby, Power system dynamics: stability and control. Chichester, U.K.: John Wiley \& Sons, 2011.

[14] P. Kundur, N. J. Balu, and M. G. Lauby, Power system stability and control. New-York, NY: McGraw-hill, 1994.

[15] P. C. Kanellakis and S. A. Smolka, "CCS expressions, finite state processes, and three problems of equivalence," Information and Computation, vol. 86, 1990

[16] "Standard 50438:2013-07: Requirements for micro-generating plants to be connected in parallel with public low-voltage distribution networks," EU Committee for Electrotechnical Standardization, Jul. 2013.

[17] C. Elbs, R. Pardatscher, R. Nenning, and R. Witzmann. (2014, Jun.) Einsatz der $\mathrm{Q}(\mathrm{U})$-regelung bei der vorarlberger energienetze $\mathrm{GmbH}$. Research Report, Technische Universitat Munchen. 